\title{
A DISTINGUISHING EXAMPLE IN $k$-SPACES
}

\author{
JOHN ISBELL
}

\begin{abstract}
Not all locally compact spaces are $k$-spaces (that is, in the coreflective hull of the compact Hausdorff spaces).
\end{abstract}

1. The largest known cartesian closed coreflective full subcategory of Top is the coreflective hull, say $\mathcal{K}_{3}$, of the category of spaces variously known as exponentiable, exponential, core-compact, or quasi-locally compact: the spaces whose topology is a continuous lattice. In $K_{3}$ is $K_{2}$, the coreflective hull of the locally compact spaces; and in $K_{2}$ is the coreflective hull $K_{1}$ of the compact Hausdorff spaces. It has been unknown whether $K_{1}=K_{3}$. This note distinguishes them; in fact, $K_{1} \neq K_{2}$. That answers Problem 5 of Herrlich [1].

Problem 6, whether $K_{2}=K_{3}$, remains. Also, the example is not sober, and thereby hangs another problem. Note that $K_{2}$ and $K_{3}$ certainly contain the same sober spaces, since every sober exponentiable space is locally compact [2].

If you want a $T_{1}$ example, help yourself, for passage to the smallest containing $T_{1}$ topology preserves local compactness and nonmembership in $K_{1}$. (The example below is locally compact; anyway, the existence of examples implies the existence of locally compact examples, since "coreflective hull" is a closure operation.)

2. What prevents compact Hausdorff spaces (and even compact normal spaces) from generating $K_{2}$ is this:

If a compact normal space has subsets $S_{\alpha}$ indexed by a non- $\sigma$-compact initial segment of the ordinals, the union of any proper initial segment of $S_{\alpha}$ 's is closed, and the union $U$ of all $S_{\alpha}$ is open, then $U$ is closed.

ProOF. Suppose $U$ is not closed. Let $x_{1}$ be a point of some $S_{\alpha_{1}}$. $I_{1}=$ $\bigcup\left[S_{\alpha}: \alpha \leq \alpha_{1}\right]$ is closed and disjoint from the closed complement $R$ of $U$, so they have disjoint neighborhoods $N_{1}, Q_{1} . N_{1} \neq U$, since $U$, being nonclosed, meets $Q_{1}$. Inductively, having $x_{1}, \ldots, x_{k}, x_{j}$ in $S_{\alpha_{j}}, x_{j+1}$ outside a neighborhood $N_{j}$ of $I_{j}$, and $N_{k} \neq U$ a neighborhood of $I_{1} \cup \cdots \cup I_{k}$, choose $x_{k+1}$ in $U-N_{k} . I_{k+1}$ and $R$ have disjoint neighborhoods, and the induction runs. But $\bigcup I_{j}$ is a countable union, hence proper and closed; it is covered by the open sets $N_{j}$, but by no finite number of them, which is absurd.

Consider the space $X$ consisting of the countable ordinals and $\omega_{1}$, with closed sets the countable initial segments, $\left\{\omega_{1}\right\}$, unions of two of those, and $X$. Every subspace is compact; for if nonempty, it has a least element, a neighborhood of which contains the rest of the subspace except perhaps $\omega_{1}$. So $X$ is locally compact. However, for any continuous map $f$ from a compact Hausdorff space to $X$, by the

Received by the editors January 27, 1986 and, in revised form, May 21, 1986.

1980 Mathematics Subject Classification. Primary 54D50.

Key words and phrases. $k$-space. 
lemma above, $f^{-1}\left(\left\{\omega_{1}\right\}\right)$ is open. Thus each such $f$ factors through $X^{*}$, which is $X$ with $\left\{\omega_{1}\right\}$ made open; and $X \in \mathcal{K}_{2}-\mathcal{K}_{1}$.

3. Let $Y$ be a topological space and $Y_{1}$ the same set with the smallest containing $T_{1}$ topology, a subbase for which is given by the open sets of $Y$ and the complements of singletons. By Alexander's Lemma, $Y_{1}$ is compact if $Y$ is. (It is a bit easier than Alexander's Lemma.) Suppose $Y$ is locally compact, and consider a basic neighborhood $W=U-\left\{x_{1}, \ldots, x_{n}\right\}$ of $p \in Y_{1}$. For $i \leq n$, if $p \notin\left\{x_{i}\right\}^{-}$in $Y$, $Y-\left\{x_{i}\right\}$ is a neighborhood of $p$; intersecting, we have $W=V-F$ where $V$ is a $y$-neighborhood of $p$ and $F$ is a (finite) set of points $x_{i}$ with $p \in\left\{x_{i}\right\}^{-}$in $Y$. Then $V$ contains a compact $(Y-)$ neighborhood $N$ of $p . N-F$ is also compact in $Y$, for any open sets of $Y$ covering it cover $p$ and $F$. By the previous remark, $N-F$ is compact in $Y_{1}$. Finally, suppose $Y_{1} \in K_{1}$. The $\mathcal{K}_{1}$-coreflection $Y^{*}$ of $Y$ has a topology contained in the topology of $Y_{1}$ (since the continuous identity function $Y_{1} \rightarrow Y$ factors through $\left.Y^{*}\right)$, and $p \in\{x\}^{-}$in $Y$ is preserved in the coreflection because of the map $[0,1] \rightarrow Y$ taking 0 to $p$ and the rest to $x$. Thus $Y^{*} \rightarrow Y$ is a homeomorphism, and $Y \in \mathcal{K}_{1}$.

\section{REFERENCES}

1. H. Herrlich, Categorical topology 1971-1981, General Topology and its Relations to Modern Analysis and Algebra $V$, Proc. Fifth Prague Topol. Sympos. 1981, Heldermann Verlag, Berlin, 1982, pp. 279-383.

2. K. H. Hofmann and J. D. Lawson, The spectral theory of distributive continuous lattices, Trans. Amer. Math. Soc. 246 (1978), 285-310.

Department of Mathematics, State University of New York at Buffalo, BUFFALO, NEW YORK 14214 